\title{
Clinical Profile and Outcome of Intracranial Bleeding in Patients Undergoing Hemodialysis at a Tertiary Hospital
}

\author{
Raviprakash Deshpande (iD ${ }^{1}$ and Chettipunyam S Chetan (id) ${ }^{2, *}$ \\ ${ }^{1}$ St John National Academy of Health Science Bengaluru, Karnataka, India \\ ${ }^{2}$ JSS Academy of Higher Education and Research, Mysore, India \\ "Corresponding author: JSS Academy of Higher Education and Research, Mysore, India. Email: cschetan_med@yahoo.co.in \\ Received 2019 September 20; Accepted 2019 December 06.
}

\begin{abstract}
Background: Stroke is one of the leading causes of mortality in the dialysis population. Although there is plenty of literature available for cardiovascular risk factors in dialysis patients, very few studies have focused on cerebrovascular events and Indian data are sparse.

Objectives: This study was undertaken to address some of these shortcomings.

Methods: This is a matched cohort study. There were 30 cases with intracranial bleeding (ICB) in hemodialysis patients whose clinical profiles were compared with 60 matched controls with no ICB and the data was analyzed.

Results: The common cause of chronic kidney disease (CKD) was diabetes. Event BP was high but not significantly. Serum albumin values were lower in patients than in controls (2.65 vs. $3.15 \mathrm{~g} / \mathrm{dL}$ ). The common site of bleeding in our study was intraparenchymal (60\%), followed by subdural hematoma (30\%). Mortality was $76.7 \%$ in one month. Although the incidence of bleeding was slightly more in the thrice-weekly dialysis group, the difference was not significant. The diabetic status of ICB patients was poorly controlled. Conclusions: Hemodialysis patients have multiple risk factors for ICB and low serum albumin could be one of them. These patients have high short-term mortality (76.7\%). There was a slightly increased incidence of ICB in patients who underwent thrice-weekly dialysis and those with poor glycemic control. This needs a systemic study with larger sample size and longer follow-up.
\end{abstract}

Keywords: Chronic Kidney Disease, Intracranial Bleeding, Hemodialysis, Heparin, Serum Albumin, Hypertension

\section{Background}

Chronic kidney disease (CKD) is increasing in prevalence in India and globally $(1,2)$. This is attributed to the increased incidence of diabetes, hypertension, and lifestylerelated factors. Raised awareness and better accessibility to health care facilities have also contributed to this high prevalence. Hemodialysis is still the predominant form of renal replacement therapy in India and many other countries (1). The patient population on dialysis is vulnerable to many complications and premature mortality due to disease per se, accelerated atherosclerosis, or dialysis treatment itself. Cardiac problems comprise major causes of mortality in dialysis patients and it has received more attention. Stroke is also a leading cause of mortality and morbidity in this dialysis population. Various studies have estimated the risk of stroke at 5 - 30 times the general population, with some showing a preponderance of ischemic stroke and some others of hemorrhagic stroke. Most data on stroke in dialysis come from Japan and the United States and the Indian data are sparse.

\section{Objectives}

The present study focused on intracranial bleeding (ICB) in dialysis patients by analyzing the clinical profile, various associated risk factors, and the impact of ICB on short-term patient survival.

\section{Methods}

This case-control study was undertaken at St. John Medical College, Bangalore, India, over a span of three years. This tertiary care hospital caters to people across all socioeconomic strata. The study included patients with CKD on long-term maintenance hemodialysis at St. Johns who either suffered ICB in the hospital or came to the emergency with the event. Those with ischemic stroke were excluded. We recorded clinical features such as vital signs, Glasgow coma score, comorbid diseases, viral serology, hemogram, blood biochemistry including liver and renal panel, and coagulation profile. The CT/MRI reports were noted for the site of ICB, the size of bleeding, and intraventricular 
extension. Dialysis records were analyzed for blood pressure readings seven and 30 days before the event, as well as laboratory reports. Thus, 30 cases with ICB were identified during the study period. The cases were well matched to the control population $(n=60)$ for demographics and duration of dialysis. Data on the controls were collected from dialysis records. Ethical committee clearance was obtained.

Summary statistics were done by proportion, mean, median, SD, and IQR. Inferential statistics were done by the chi-square test, independent t test, and Mann-Whitney test. All measurements were done using SPSS 16.0.

\section{Results}

Males outnumbered females in our study (69.5\% vs. $30.5 \%$ ). The average age of the study population was 53.48 years. Moreover, $92.7 \%$ of the study population was hypertensive (cases $95.7 \%$, controls $91.3 \%$ ) and $60.87 \%$ of the study population was diabetic (cases $65.2 \%$, controls $58.7 \%$ ) (Table 1).

Diabetes was the most common cause of CKD, accounting for $62.3 \%$ (cases $56.5 \%$, controls $65.2 \%$ ), followed by chronic glomerulonephritis (CGN) (Table 1). An average of 28,527 dialysis sessions is conducted annually at our center; 720 patients were on maintenance hemodialysis during the study period, of whom 30 suffered ICB (prevalence $3.19 \%)$.

About 57.8\% (52/90 patients) of our study patients were on thrice-weekly dialysis and the rest was on twice a week dialysis (38/90 patients). All patients underwent dialysis sessions for four hours, with a P value of 0.07 for the frequency of dialysis between cases and controls (Table 1). In patients with ICB, the mean duration of dialysis was 21.78 months with a range of 1 - 72 months (cases and controls were matched for the duration of dialysis).

The average dose of unfractionated heparin used was 90.36 units per $\mathrm{kg}$ body weight in cases and 86.48 units per kg among controls $(\mathrm{P}=0.5$ ). Fifteen (out of 28) patients with ICB were using antiplatelet at the time of the event $(\mathrm{P}=0.6)$. Prothrombin time $(\mathrm{PT})$ and activated partial thromboplastin time (APTT) done after the event were not deranged in cases. Platelet counts were available in both groups and were not different between the two groups (Table 1). The BP recordings at the time of stroke (current) and $B P$ readings in the preceding one week and one month were analyzed, which showed no statistically significant difference (Table 2).

Serum albumin at the time of stroke and prior albumin values were significantly lower in those with ICB than in controls (2.65 vs. $3.15, \mathrm{P}=0.005$ at the event; 2.70 vs. 3.07, $\mathrm{P}$
$=0.036$ prior to the event). Serum sodium, potassium, calcium, phosphorous, and liver function tests were not different between cases and controls. Random blood glucose levels (RBS) were slightly high in cases $(\mathrm{P}=0.04)$ (Table 3$)$.

the most common site of bleeding was intraparenchymal (60\%) followed by Sub dural hematoma (30\%). Three patients had primary intraventricular bleeding. Clinical features like vomiting, altered sensorium, lateralizing signs, and seizures could not predict the site of ICB. The mean time to occurrence of ICB event was 2 hours from last heparin dose (range 0 - 24 hours; five patients had ICB while dialysis was in process, the rest had it after dialysis was completed). There was a history of trauma in $30 \%$ of the cases, old CVA in $21.7 \%$, evidence of micro bleeding in $14.3 \%$, and lacunar infarcts in $9.5 \%$ (Table 4 ).

There were 23 deaths in the first one month and the cause was probably brain stem dysfunction and herniation; two patients succumbed to septic shock.

\section{Discussion}

The prevalence of CKD is increasing in India and globally. All stages of CKD are associated with high morbidity and mortality. This risk, which begins in early CKD, increases as CKD advances (3). Coronary artery disease and stroke are the leading causes of mortality in the chronic dialysis population. The incidence of stroke in dialysis patients varies from 10 to 33 per 1000 patient-years and is 5 - 30 times greater than in the general population. The age-adjusted relative risk of stroke among dialysis patients compared to the general population was 6.1 for Caucasians and 9.7 for African-American males (4). Patients on dialysis had a higher incidence of hemorrhagic stroke than the general population. A 22-year single-center study of stroke in dialysis patients noticed that stroke occurred at a younger age ( $64 \pm 10$ vs. $67 \pm 13$ years) and hemorrhagic stroke was more common (52\%) than ischemic stroke $(4,5)$.

Risk factors for stroke include modifiable and nonmodifiable factors such as the old age, non-Caucasian and Asian ethnicity, and family history. Diabetes and hypertension have emerged as major risk factors for ischemic and hemorrhagic stroke (6). Chronic kidney disease has a higher burden of traditional risk factors and nontraditional risk factors like hyper-homocysteinemia, hyperuricemia, chronic inflammation, asymmetric dimethylarginine, oxidative stress, anemia, thrombogenic factors, endothelial dysfunction, arterial stiffness, and impaired cerebral auto regulation (7). Patients with CKD and stroke have shown an increased prevalence of intracranial artery calcification from 76.2 to $95 \%$ in various studies (8). Pulse wave velocity, which reflects arterial stiffness, is high in 


\begin{tabular}{|c|c|c|c|}
\hline & ICB $(N=30$ Cases $)$ & No ICB $(N=60$ Controls $)$ & $\mathbf{P}$ \\
\hline Age & $53.48 \pm 11.56$ & $52.96 \pm 10.50$ & 0.85 \\
\hline Gender & & & 0.6 \\
\hline Male & $20(66.7)$ & $43(71.7)$ & \\
\hline Female & $10(33.3)$ & $17(28.3)$ & \\
\hline Systemic hypertension & $28(93.33)$ & $55(91.7)$ & 0.5 \\
\hline Diabetes & $20(66.7)$ & $35(58.3)$ & 0.6 \\
\hline HbS Ag-positive & $1(3.33)$ & $8(13.33)$ & 0.07 \\
\hline Hepatitis C-positive & $4(13.33)$ & $17(28.33)$ & 0.2 \\
\hline HIV-positive & $2(6.7)$ & $2(3.33)$ & 0.2 \\
\hline Pulmonary Tuberculosis & $4(13.33)$ & $5(8.33)$ & 0.3 \\
\hline Past CVA & $4(13.3)$ & 0 & \\
\hline Frequency of dialysis & & & 0.06 \\
\hline Two per week & $8(26.7)$ & $30(50)$ & \\
\hline Three per week & $22(73.33)$ & $30(50)$ & \\
\hline Heparin (anticoagulation during dialysis) & & & 0.06 \\
\hline Systemic heparin & $2(6.7)$ & $2(3.3)$ & \\
\hline Tight heparin & $26(86.7)$ & $58(96.7)$ & \\
\hline Low-molecular weight heparin & $0(0)$ & $0(0)$ & \\
\hline Saline & $2(6.7)$ & $0(0)$ & \\
\hline Heparin average Dose & $90.36 \pm 24.8$ & $86.48 \pm 12.9$ & 0.5 \\
\hline Major causes of CKD & & & 0.09 \\
\hline Diabetic nephropathy & $17(56.7)$ & $39(65.0)$ & \\
\hline Chronic glomerular & $11(36.7)$ & $13(21.7)$ & \\
\hline Chronic interstitial disease & $2(6.7)$ & $5(8.33)$ & \\
\hline Chronic allograft nephropathy & $0(0)$ & $3(5)$ & \\
\hline Platelet Count (lakhs/microliter) & $17738 \pm 807274$ & $217570 \pm 73324$ & 0.06 \\
\hline Aspirin Use & $5(16.7)$ & $17(28.3)$ & 0.7 \\
\hline Clopidogrel Use & $6(20.0)$ & $8(13.3)$ & 0.7 \\
\hline Aspirin + clopidogrel & $4(13.3)$ & $12(20.0)$ & 0.7 \\
\hline No antiplatelets & $15(50)$ & $23(38.33)$ & 0.7 \\
\hline
\end{tabular}

${ }^{\mathrm{a}}$ Values are expressed as No. (\%) or mean $\pm \mathrm{SD}$.

CKD. Vascular calcification and stiffness can worsen hypertension and together with anticoagulation may increase the risk of hemorrhagic stroke (9). Anemia, which invariably occurs as CKD progresses, leads to the increased stroke risk, mostly ischemic stroke (10), but may also result in bigger hematomas as shown by studies of critically ill patients (11) although not much literature is available on the dialysis population.

Dialysis patients with hemorrhagic stroke had poor survival and high mortality in our study, which was $76.7 \%$ at one month that is similar to other studies showing 53\% $79 \%$ mortality (10-12). Mortality is highest in the first three months and is much higher than the mortality of patients with cerebral infarction (11-13). In our study, most deaths occurred either immediately or within three months of the event. Patients who suffered ICB were on dialysis for a longer duration than those who suffered cerebral infarction; in our study, 39.1\% had been on dialysis for three years or more. Patients who present the reduced levels of consciousness on admission have larger hematoma and irreg- 


\begin{tabular}{|c|c|c|c|}
\hline & ICB (Cases) & No ICB (Controls) & $\mathbf{P}$ \\
\hline Mid dialysis SBP, mmHg (on the day of the event or in the last HD before the event) & $148.57 \pm 19.82$ & $146.30 \pm 18.1$ & 0.7 \\
\hline Mid DBP Dialysis, mmHg (on the day of the event or in the last HD before the event) & $84.29 \pm 7.46$ & $84.35 \pm 6.2$ & 0.9 \\
\hline Pre-dialysis SBP, mmHg (on the day of the event or in the last HD before the event) & $145.45 \pm 16.83$ & $143.9 \pm 17.1$ & 0.7 \\
\hline Pre-dialysis DBP, $\mathrm{mmHg}$ (on the day of the event or in the last HD before the event) & $84.36 \pm 7.03$ & $84.6 \pm 6.4$ & 0.9 \\
\hline Seven-day prior SBP, mmHg & $151.74 \pm 18.25$ & $143.95 \pm 17.9$ & 0.1 \\
\hline Seven-day prior DBP, $\mathrm{mmHg}$ & $87.65 \pm 7.95$ & $85.58 \pm 5.5$ & 0.2 \\
\hline 30-day Prior SBP, mmHg & $146.36 \pm 13.99$ & $141.46 \pm 17.1$ & 0.8 \\
\hline 30-day Prior DBP, mmHg & $85.91 \pm 7.34$ & $85.54 \pm 5.9$ & 0.2 \\
\hline
\end{tabular}

${ }^{\mathrm{a}}$ Values are expressed as mean $\pm \mathrm{SD}$.

\begin{tabular}{|c|c|c|c|}
\hline & Cases & Controls & $\mathbf{P}$ \\
\hline Serum Protein current & $6.51 \pm 1.11$ & $7.00 \pm 1.03$ & 0.08 \\
\hline Serum protein prior & $6.63 \pm 0.80$ & $6.77 \pm 0.93$ & 0.5 \\
\hline Albumin current & $2.65 \pm 0.60$ & $3.15 \pm 0.7$ & 0.005 \\
\hline Albumin prior & $2.70 \pm 0.62$ & $3.07 \pm 0.7$ & 0.036 \\
\hline B urea current & $103(60-132)$ & $80(57-119)$ & 0.6 \\
\hline B urea prior & $112(60-132)$ & $124(75-147)$ & 0.7 \\
\hline Na current & $135.09 \pm 3.9$ & $135.00 \pm 4.7$ & 0.5 \\
\hline Na prior & $133.8 \pm 5.65$ & $135.60 \pm 3.7$ & 0.2 \\
\hline $\mathrm{K}^{+}$current & $4.56 \pm 0.99$ & $4.82 \pm 0.9$ & 0.9 \\
\hline $\mathbf{K}^{+}$prior & $4.71 \pm 0.63$ & $4.83 \pm 0.9$ & 0.1 \\
\hline Calcium current & $8.33 \pm 1.58$ & $8.53 \pm 0.7$ & 0.3 \\
\hline Calcium prior & $8.67 \pm 1.13$ & $8.34 \pm 0.8$ & 0.6 \\
\hline Phosphorous current & $4.3(2.7-5.7)$ & $4.4(3.4-5.3)$ & 0.3 \\
\hline Phosphorous prior & $4(3.1-4.8)$ & $4.9(3.9-6.4)$ & 0.8 \\
\hline Random blood sugar current & $180.70 \pm 73.56$ & $142.03 \pm 69.9$ & 0.04 \\
\hline HB\% current & $9.38 \pm 1.79$ & $9.37 \pm 1.7$ & 0.9 \\
\hline HB\% prior & $9.03 \pm 1.81$ & $9.66 \pm 1.6$ & 0.1 \\
\hline AST current & $30(20-55)$ & $24(16-39.5)$ & 0.9 \\
\hline AST prior & $20(18-28)$ & $21.5(14.5-30)$ & 0.09 \\
\hline ALT current & $35(22-64)$ & $33.5(24-54.5)$ & 0.9 \\
\hline ALT prior & $29(24-60)$ & $29.5(24-44.5)$ & 0.8 \\
\hline
\end{tabular}

${ }^{\mathrm{a}}$ Values are expressed as median (IQR) or mean $\pm \mathrm{SD}$. 


\begin{tabular}{|c|c|}
\hline & Values \\
\hline Number of ICB cases presenting with vomiting & $20(65.2)$ \\
\hline Number of ICB cases presenting with altered sensorium & $20(65.2)$ \\
\hline Number of ICB cases with lateralizing deficits & $11(35.0)$ \\
\hline Number of ICB cases with seizures & $3(10)$ \\
\hline GCS on admission & $7(3-14)$ \\
\hline \multicolumn{2}{|l|}{ Site of bleeding } \\
\hline Intraparenchymal & $18(60)$ \\
\hline Subdural & $9(30)$ \\
\hline Subarachnoid hemorrhage & $3(10)$ \\
\hline Past history of CVA & $4(13.33)$ \\
\hline Past history of IHD & $3(10.0)$ \\
\hline Time to event from last heparin dose, $h$ & $2(0-24)$ \\
\hline Micro bleed (yes) & $4(13.33)$ \\
\hline Lacunar infarct (yes) & $3(10.0)$ \\
\hline Number of ICB patients on erythropoietin & $8(26.7)$ \\
\hline Trauma history & $9(30.4)$ \\
\hline \multicolumn{2}{|l|}{ Outcome of ICB by day $30^{b}$} \\
\hline \multicolumn{2}{|l|}{ Complete recovery to normal $(\mathrm{n}=0)$} \\
\hline Partial recovery $(n=7)$ & $14(10-15)$ \\
\hline Death $(n=23)$ & $3.5(3-13)$ \\
\hline
\end{tabular}

Abbreviations: CVA, cerebrovascular accident; IHD, ischemic heart disease. ${ }^{\mathrm{a}}$ Values are expressed as No. (\%) or median (IQR).

${ }^{\mathrm{b}} \mathrm{P}=0.1$ in Mann-Whitney test.

ular hematoma and often reveal hematoma enlargement on repeat CT scans $(14,15)$. Those with large hematomas, especially more than $60 \mathrm{~mL}$, and pontine hematoma showed a poor outcome (16). In the current study, we did not systematically analyze the hematoma volume and its relationship with the outcome. Abnormal coagulation, such as prolonged prothrombin time and fibrin degradation products, is seen in some studies (17) whereas no significant correlation was seen between fibrinogen levels and hematoma size in others (18). In our study, there was no significant difference in the coagulation profile as made out by platelet counts within the normal range, APTT, PT, bleeding time, and clotting time. Some studies reported the most common site of bleeding in dialysis patients is the basal ganglia and ventricles (19); in our study, it was intraparenchymal, followed by subdural hematoma.

Hypertension is a risk factor for ICB. In CKD, blood pressure (BP) is difficult to control and often requires multiple antihypertensive medications. Poorly controlled BP after an ICB event can increase the risk of re-bleeding and worsen the neurological outcomes. The strict control of
$\mathrm{BP}$ is required to reduce the size of intracerebral bleeding and improve the prognosis following a bleeding event (1618). What correlates better with the outcome, initial BP or later $\mathrm{BP}$ recordings, systolic BP or diastolic BP, is subject to debate. Kim et al.'s (20) study found no relationship with the initial BP but the third day BP was correlated with outcome. In our study, we did not observe a statistically significant difference in current BP or prior BP and the BP of the case group was not different from that of the selected control group. This does not undermine the importance of BP control; however, many of our patients were on multiple antihypertensive medications. The most common cause of death in ICB patients is brain stem herniation, which is the result of raised intracranial tension due to either hematoma per se or cerebral edema caused by dialysis disequilibrium as a result of rapid changes in osmolality with intermittent therapies (21).

Malnutrition is a well-recognized problem in hemodialysis patients. Its causes are multifactorial, including increased common catabolism and decreased intake $(12,17)$. They are due to the increased levels of pro-inflammatory cytokines in CKD and dialysis patients. The malnutrition inflammation complex leads to poor outcomes and increases all-cause mortality (22). Undernutrition is associated with a higher risk of ICB in dialysis patients. This is in contrast to the general population where obesity confers a higher stroke risk. Low albumin affecting erythrocyte deformability and endothelial dysfunction may well explain the increased stroke risk in malnourished patients $(23,24)$. The observation of low albumin in our study concurs with the available literature and emphasizes the importance of maintaining good nutrition in dialysis patients. The control group without ICB had higher serum albumin levels.

Heparin is the most commonly used anticoagulant for hemodialysis (25). It is used as an initial bolus, followed by hourly boluses, with the last dose given one hour prior to the termination of dialysis. Although it potentially causes serious bleeding complications, in view of its short halflife of 30 minutes to two hours, bleeding episodes related to intracranial, gastrointestinal and pericardium hemorrhages are not common (26). The safety of low-molecularweight heparin has been proven by many studies though its anticoagulant effect lasts four hours $(27,28)$. Heparin on a background of uremic bleeding diathesis could lead to an increased risk of hemorrhagic stroke (29). If intracerebral bleeding does occur in a dialysis patient, systemic heparinization can increase the hematoma size (30) and worsen the outcome. The use of aspirin was associated with increased intracerebral bleeding in a study and did not confer significant benefit to cardiovascular outcome (31). Our current study showed no significant difference in 
the average heparin dose and antiplatelet use between the case and control groups.

Diabetes may increase the vascular risk but its role is not clear in the etiopathogenesis of spontaneous intracranial hemorrhage, with some studies showing an positive association, others not, and even some studies showing a reduced incidence of bleeding in the general population (32-34). Poor glycemic control confers increased mortality in hospitalized patients. Our study patients had higher blood sugar at the time of the event than the controls.

In conclusion, hemodialysis patients have multiple risk factors for ICB. Blood pressure was high during the event although it did not achieve statistical significance. Optimal control of BP is important to reduce ICB and its complications. The common site of bleeding was intraparenchymal, followed by SDH. There was a higher incidence of ICB, although not statistically significant, in patients on thrice-weekly dialysis. Low serum albumin, which is an indicator of poor nutritional status, carried a higher risk for ICB; thus, it is important to maintain a good nutrition status in dialysis patients. Patients with ICB had high mortality and most deaths (76.7\%) occurred within one month of the event.

One of the main limitations of our study is the sample size. Larger sample size would have better addressed the role of many factors including uncontrolled glycemic state, hypertension, and thrice-weekly dialysis in increased predisposition to ICB in the dialysis population.

\section{Footnotes}

Authors' Contribution: Hemodialysis patients are at higher risk for Intracranial bleed compared to general population and mortality is very high in this patient group.

Ethical Approval: Clearance was obtained from the St. John Medical College Institutional Ethical Committee (IERB: 222/2010).

\section{Funding/Support: None.}

\section{References}

1. Varughese S, Abraham G. Chronic kidney disease in India: A clarion call for change. Clin J Am Soc Nephrol. 2018;13(5):802-4. doi: 10.2215/CJN.09180817. [PubMed: 29382651]. [PubMed Central: PMC5969474].

2. Varma PP. Prevalence of chronic kidney disease in India - where are we heading? Indian J Nephrol. 2015;25(3):133-5. [PubMed: 26060360]. [PubMed Central: PMC4446915].

3. Shamseddin MK, Parfrey PS. Sudden cardiac death in chronic kidney disease: Epidemiology and prevention. Nat Rev Nephrol. 2011;7(3):14554. doi: 10.1038/nrneph.2010.191. [PubMed: 21283136].

4. Nayak-Rao S, Shenoy MP. Stroke in patients with chronic kidney disease...: How do we approach and manage it? Indian J Nephrol.
2017;27(3):167-71. doi: 10.4103/0971-4065.202405. [PubMed: 28553032] [PubMed Central: PMC5434678].

5. Toyoda K, Fujii K, Fujimi S, Kumai Y, Tsuchimochi H, Ibayashi $S$, et al. Stroke in patients on maintenance hemodialysis: A 22 year single-center study. Am J Kidney Dis. 2005;45(6):1058-66. doi: 10.1053/j.ajkd.2005.02.028. [PubMed: 15957135].

6. Power A. Stroke in dialysis and chronic kidney disease. Blood Purif. 2013;36(3-4):179-83. doi: 10.1159/000356086. [PubMed: 24496188].

7. Townsend RR. Stroke in chronic kidney disease: prevention and management. Clin J Am Soc Nephrol. 2008;3 Suppl 1:S11-6. doi: 10.2215/CJN.03260807. [PubMed: 18178791]. [PubMed Central: PMC3152267].

8. Bugnicourt JM, Chillon JM, Massy ZA, Canaple S, Lamy C, Deramond $\mathrm{H}$, et al. High prevalence of intracranial artery calcification in stroke patients with CKD: A retrospective study. Clin J Am Soc Nephrol. 2009;4(2):284-90. doi: 10.2215/CJN.02140508. [PubMed: 19158370]. [PubMed Central: PMC2637585].

9. Sozio SM, Armstrong PA, Coresh J, Jaar BG, Fink NE, Plantinga LC, et al. Cerebrovascular disease incidence, characteristics, and outcomes in patients initiating dialysis: the choices for healthy outcomes in caring for ESRD (CHOICE) study. Am J Kidney Dis. 2009;54(3):468-77. doi: 10.1053/j.ajkd.2009.01.261. [PubMed: 19376618]. [PubMed Central: PMC2744381].

10. Abramson JL, Jurkovitz CT, Vaccarino V, Weintraub WS, McClellan W. Chronic kidney disease, anemia, and incident stroke in a middle-aged, community-based population: The ARIC Study. Kidney Int. 2003;64(2):610-5. doi: 10.1046/j.1523-1755.2003.00109.x. [PubMed: 12846757].

11. Kumar MA, Rost NS, Snider RW, Chanderraj R, Greenberg SM, Smith EE, et al. Anemia and hematoma volume in acute intracerebral hemorrhage. Crit Care Med. 2009;37(4):1442-7. doi: 10.1097/CCM.0b013e31819ced3a. [PubMed: 19242340].

12. Iseki K, Kawazoe N, Osawa A, Fukiyama K. Survival analysis of dialysis patients in Okinawa, Japan (1971-1990). Kidney Int.1993;43(2):404-9. doi: 10.1038/ki.1993.59. [PubMed: 8441236].

13. Lin CY, Chien CC, Chen HA, Su FM, Wang JJ, Wang CC, et al The impact of comorbidity on survival after hemorrhagic stroke among dialysis patients: A nationwide population-based study. BMC Nephrol. 2014;15:186. doi:10.1186/1471-2369-15-186. [PubMed: 25427630]. [PubMed Central: PMC4256891].

14. Kawahata N. Brain hemorrhage associated with maintenance hemodialysis. CT analysis of 19 cases. Nosotchu. 1994;16(2):79-86. doi: 10.3995/jstroke.16.79.

15. Murakami M, Hamasaki T, Kimura S, Maruyama D, Kakita K. Clinical features and management of intracranial hemorrhage in patients undergoing maintenance dialysis therapy. Neurol Med Chir (Tokyo). 2004;44(5):225-32. discussion 233. doi: 10.2176/nmc.44.225. [PubMed: 15200056].

16. Shimojo S, Ebisawa T, Hasegawa T, Toyohara K, Sakai O. Intracranial hemorrhage in chronic renal failure. Nosotchu. 1997;19(1):66-70. doi 10.3995/jstroke.19.66

17. Fujii Y, Takeuchi S, Sasaki O, Minakawa T, Tanaka R. Multivariate analysis of predictors of hematoma enlargement in spontaneous intracerebral hemorrhage. Stroke. 1998;29(6):1160-6. doi: 10.1161/01.str.29.6.1160. [PubMed: 9626289].

18. Miyahara K, Murata H, Abe H. Predictors of intracranial hematoma enlargement in patients undergoing hemodialysis. Neurol Med Chir (Tokyo). 2007;47(2):47-51. discussion 51-2. doi: 10.2176/nmc.47.47. [PubMed: 17317940].

19. Sakamoto N, Ishikawa E, Aoki K, Uemae Y, Komatsu Y, Matsumura A. Clinical outcomes of intracerebral hemorrhage in hemodialysis patients. World Neurosurg. 2014;81(3-4):538-42. doi: 10.1016/j.wneu.2013.10.033. [PubMed: 24141001].

20. Kim JK, Shin JJ, Park SK, Hwang YS, Kim TH, Shin HS. Prognostic factors and clinical outcomes of acute intracerebral hemorrhage in patients 
with chronic kidney disease. J Korean Neurosurg Soc. 2013;54(4):296301. doi: 10.3340/jkns.2013.54.4.296. [PubMed: 24294452]. [PubMed Central: PMC3841271].

21. Ochiai H, Uezono S, Kawano H, Ikeda N, Kodama K, Akiyama H. Factors affecting outcome of intracerebral hemorrhage in patients undergoing chronic hemodialysis. Ren Fail. 2010;32(8):923-7. doi 10.3109/0886022X.2010.502279. [PubMed: 20722558].

22. Kalantar-Zadeh K, Block G, McAllister CJ, Humphreys MH, Kopple JD. Appetite and inflammation, nutrition, anemia, and clinical outcome in hemodialysis patients. Am J Clin Nutr. 2004;80(2):299-307. doi: 10.1093/ajcn/80.2.299. [PubMed: 15277149].

23. Krishna PR, Naresh S, Krishna GS, Lakshmi AY, Vengamma B, Kumar VS. Stroke in chronic kidney disease. Indian J Nephrol. 2009;19(1):5-7. doi: 10.4103/0971-4065.50672. [PubMed: 20352003]. [PubMed Central: PMC2845195].

24. Saeed F, Kousar N, Qureshi K, Laurence TN. A review of risk factors for stroke in patients with chronic kidney disease.J Vasc Interv Neurol. 2009;2(1):126-31. [PubMed: 22518239]. [PubMed Central: PMC3317334].

25. Sonawane S, Kasbekar N, Berns JS. The safety of heparins in end stage renal disease. Semin Dial. 2006;19(4):305-10. doi: 10.1111/j.1525139X.2006.00177.x. [PubMed: 16893408].

26. Chen HH, Hsu CC, Weng SF, Lin HJ, Wang JJ, Guo HR, et al. Hemodialysis with end-stage renal disease did not raise the risk of intracranial hemorrhage after a head injury. Scand J Trauma Resusc Emerg Med. 2015;23:82. doi: 10.1186/s13049-015-0168-1. [PubMed: 26511383]. [PubMed Central: PMC4625736].

27. Lai S, Barbano B, Cianci R, Gigante A, Di Donato D, Asllanaj B, et al. [The risk of bleeding associated with low molecular weight heparin in patients with renal failure]. G Ital Nefrol. 2010;27(6):649-54. Peersian. [PubMed: 21132647].
28. Lim W, Cook DJ, Crowther MA. Safety and efficacy of low molecular weight heparins for hemodialysis in patients with end-stage renal failure: A meta-analysis of randomized trials. J Am Soc Nephrol. 2004;15(12):3192-206. doi: 10.1097/01.ASN.0000145014.80714.35. [PubMed: 15579523].

29. Seliger SL, Gillen DL, Longstreth WJ, Kestenbaum B, StehmanBreen CO. Elevated risk of stroke among patients with end-stage renal disease. Kidney Int. 2003;64(2):603-9. doi: 10.1046/j.15231755.2003.00101.x. [PubMed: 12846756].

30. Onoyama K, Ibayashi S, Nanishi F, Okuda S, Oh Y, Hirakata H, et al. Cerebral hemorrhage in patients on maintenance hemodialysis. CT analysis of 25 cases. EurNeurol.1987;26(3):171-5. doi: 10.1159/000116330. [PubMed: 3569371].

31. Aoun M, Koubar SH, Antoun L, Tamim H, Makki M, Chelala D. Reduction of intracerebral hemorrhage in hemodialysis patients after reducing aspirin use: A quality-assurance observational study. PLoS One. 2017;12(10). e0185847. doi: 10.1371/journal.pone.0185847. [PubMed: 28968454]. [PubMed Central: PMC5624631].

32. Emerging Risk Factors C, Sarwar N, Gao P, Seshasai SR, Gobin $\mathrm{R}$, Kaptoge S, et al. Diabetes mellitus, fasting blood glucose concentration, and risk of vascular disease: A collaborative metaanalysis of 102 prospective studies. Lancet. 2010;375(9733):2215-22. doi: 10.1016/S0140-6736(10)60484-9. [PubMed: 20609967]. [PubMed Central: PMC2904878].

33. Inagawa T. Risk factors for primary intracerebral hemorrhage in patients in Izumo City, Japan. Neurosurg Rev. 2007;30(3):225-34. discussion 234. doi: 10.1007/s10143-007-0082-8. [PubMed: 17503099].

34. Jorgensen H, Nakayama H, Raaschou HO, Olsen TS. Stroke in patients with diabetes. The Copenhagen Stroke study. Stroke. 1994;25(10):197784. doi: 10.1161/01.str.25.10.1977. [PubMed: 8091441]. 